\title{
Reinforcement of calcium phosphate cement using alkaline-treated silk fibroin
}

This article was published in the following Dove Press journal:

International Journal of Nanomedicine

\author{
Muli $\mathrm{Hu}^{1,2, *}$ \\ Zhiwei $\mathrm{He}^{2,3, *}$ \\ Fengxuan $\operatorname{Han}^{1,2}$ \\ Chen $\mathrm{Shi}^{4}$ \\ Pinghui Zhou ${ }^{2}$ \\ Feng Ling ${ }^{1,2}$ \\ Xuesong Zhu ${ }^{2}$ \\ Huilin Yang ${ }^{2}$ \\ Bin $\mathrm{Li}^{2,5}$
}

'Department of Polymer Science, College of Chemistry, Chemical

Engineering and Materials Science, Orthopaedic Institute, Soochow

University, Suzhou, China;

${ }^{2}$ Department of Orthopaedic Surgery,

The First Affiliated Hospital of Soochow University, Suzhou, China; ${ }^{3}$ Department of Orthopaedics, Jinling Hospital, School of Medicine, Nanjing University, Nanjing, China; ${ }^{4}$ Department of Biomedical Engineering, National University of Singapore, Singapore, Singapore;

${ }^{5}$ China Orthopaedic Regenerative

Medicine Group (CORMed),

Hangzhou, China

*These authors contributed equally to this work
Correspondence: Bin $\mathrm{Li}$

Department of Orthopaedic Surgery,

The First Affiliated Hospital of Soochow University, 708 Renmin Rd, Rm 308

Bldg I, Soochow University (South Campus), Suzhou, Jiangsu 215007, China

Tel +865I26778 II 63

Email binli@suda.edu.cn
Background: Bone cement plays an important role in the treatment of osteoporotic vertebral compression fractures. Calcium phosphate cement (CPC) is a potential alternative to poly(methyl methacrylate), currently the gold standard of bone cements. However, the poor mechanical properties of CPCs limit their clinical applications. The objective of this study was to develop reinforced CPCs for minimally invasive orthopedic surgeries by compositing silk fibroin (SF) with $\alpha$-tricalcium phosphate.

Methods: SF solution was treated with calcium hydroxide and characterized by Zeta potential analyzer and Fourier transform infrared spectroscopy. The alkaline-treated SF (tSF) was composited with $\alpha$-tricalcium phosphate to obtain tSF/CPC composite, which was characterized using mechanical tests, scanning electron microscopy, handling property and biocompatibility tests, and sheep vertebral augmentation tests.

Results: Upon treatment with calcium hydroxide, larger SF particles and more abundant negative charge appeared in tSF solution. The tSF/CPCs exhibited a compact structure, which consisted of numerous SF -CPC clusters and needle-like hydroxyapatite (HAp) crystals. In addition, high transition rate of HAp in tSF/CPCs was achieved. As a result, the mechanical property of tSF/ $\mathrm{CPC}$ composite cements was enhanced remarkably, with the compressive strength reaching as high as $56.3 \pm 1.1 \mathrm{MPa}$. Moreover, the $\mathrm{tSF} / \mathrm{CPC}$ cements showed good injectability, anti-washout property, and decent biocompatibility. The tSF/CPCs could be used to augment defected sheep vertebrae to restore their mechanical strength.

Conclusion: $\mathrm{tSF} / \mathrm{CPC}$ may be a promising composite bone cement for minimally invasive orthopedic surgeries.

Keywords: bone cement, silk fibroin, calcium phosphate, calcium hydroxide, reinforcement

\section{Introduction}

Poly(methyl methacrylate) (PMMA) bone cements are commonly used for prosthesis fixation or vertebral augmentation in orthopedics surgeries, such as joint replacement, vertebroplasty (VP), and kyphoplasty (KP). However, PMMA bone cements have intrinsic limitations, including monomer toxicity, nonbiocompatibility, and excessive heat generation upon solidification. ${ }^{1}$ Alternatively, calcium phosphate cements (CPCs), first invented in the 1980s, have received increasing attention as a type of biodegradable and biocompatible bone grafting and filling material. ${ }^{2}$ Recently, minimally invasive surgeries, such as VP and KP, in which high-strength bone cements are needed, have been developed to relieve the morbidity of vertebral compression fractures. ${ }^{1,3}$ However, due to the poor mechanical properties of CPCs, their applications in orthopedic surgeries at such high load-bearing regions are largely limited. ${ }^{4}$

To date, many approaches, including porosity reduction, fiber reinforcement, and dual setting, have been sought in order to improve the mechanical properties of CPCs. ${ }^{5}$ The porosity reduction of CPCs is based on the optimization of an intrinsic cement 
property by changing the liquid/powder ratio, which may adversely affect the handling property of cement. ${ }^{6-9}$ Both fiber reinforcement and dual setting require mixing of $\mathrm{CPC}$ with other components. ${ }^{10-12}$ Some synthetic polymers, such as carbon nanotubes and poly(lactide-co-glycolide) (PLGA) and natural polymers, such as chitosan and cellulose have been shown to enhance the mechanical properties of CPCs. By controlling the growth of crystals of hydroxyapatite (HAp) in CPCs, such polymers can affect the conversion rate and HAp crystal types and as a result, strengthen CPCs. ${ }^{13-17}$ However, the weak interfacial adhesion between the organic and inorganic phases still remains a major concern, which limits further enhancement of CPCs. ${ }^{18}$ Hence, a number of studies have attempted to modify these fillers to ameliorate interfacial bonding between the 2 phases. ${ }^{19,20}$

Silk fibroin (SF), a popular material with good biocompatibility, is a promising biomaterial for biomedical applications. ${ }^{20}$ Previously, SF has been used in CPCs by various groups, including us, to improve their mechanical properties. $^{21-25}$ The $-\mathrm{COOH}$ and $-\mathrm{OH}$ groups on SF can react with $\mathrm{Ca}^{2+}$ from tricalcium phosphate (TCP) and form nuclear sites to grow HAp. ${ }^{15,19} \mathrm{pH}$ also affects HAp formation by regulating TCP hydration and SF structure. ${ }^{20,21}$ Attempts have also been made to use SF-HAp complex, acidified SF, and $N$-acetyl cysteine to enhance the interfacial bonding between SF and CPCs. ${ }^{24-26}$ However, their final mechanical performance is still insufficient for clinical needs.

In this study, we proposed to use SF treated with calcium hydroxide, referred to here as $\mathrm{tSF}$, as curing liquid for $\alpha$-TCP to prepare $\mathrm{tSF} / \mathrm{CPC}$ composite bone cements. We hypothesized that after treatment with calcium hydroxide, the increased number of negative charges on tSF chains could provide more nuclear sites and facilitate HAp formation after mixing with $\alpha$-TCP. The handling property, setting time, mechanical properties, and biocompatibility of tSF/ CPC composite bone cements were determined.

\section{Materials and methods} Preparation of $\mathrm{tSF}$ solution

Bombyx mori (silkworm) SF solution was prepared according to a published protocol. ${ }^{27}$ The SF solution was condensed to $20 \mathrm{wt} \%$ using polyethylene glycol (>99\%, Sinopharm Chemical Reagent, Shanghai, China) solution. Then the condensed SF solution was treated with saturated calcium hydroxide (99\%, Sinopharm Chemical Reagent) solution. In brief, a certain amount of saturated clarification limewater was added to $5 \mathrm{~mL}$ condensed $\mathrm{SF}$ solution to reach a final $\mathrm{pH}$ of 8.0 , 8.5, 9.0, and 9.5. After being placed in the oven for 4 hours, the treated SF solutions were named as $8 \mathrm{tSF}, 8.5 \mathrm{tSF}, 9 \mathrm{tSF}$, and 9.5tSF, respectively. An untreated SF solution $(\mathrm{pH}=7.5)$ named SF was used as a control group. For CPCs without SF, PBS solution or saturated limewater-modified PBS with different $\mathrm{pH}(8.0,8.5$, and 9.0) was also prepared.

\section{Preparation of CPCs}

The $\alpha$-TCP (99\%, Alfa Aesar, Ward Hill, MA, USA) powder was mixed with curing liquid for 2 minutes until a paste was formed. CPC was prepared using PBS as curing liquid. Alkaline (ALK)/CPCs prepared using PBS at $\mathrm{pH}$ values of $8.0,8.5$, and 9.0 as curing liquids, were named $8 \mathrm{ALK} /$ $\mathrm{CPC}, 8.5 \mathrm{ALK} / \mathrm{CPC}$, and 9ALK/CPC, respectively. SF/CPC was obtained with $\mathrm{SF}$ solution, and $8 \mathrm{tSF} / \mathrm{CPC}, 8.5 \mathrm{tSF} / \mathrm{CPC}$, and $9 \mathrm{tSF} / \mathrm{CPC}$ were separately prepared using $8 \mathrm{tSF}, 8.5 \mathrm{tSF}$, and $9 \mathrm{tSF}$ solutions. The compositions of various CPCs are shown in Table 1.

\section{Characterizations}

The particle size and zeta potential of SF and tSF solutions were measured by Zetasizer Nano ZS90. SF and 8.5tSF solutions were freeze-dried by Freeze Drier (Christ Alpha1-2) for Fourier transform infrared spectroscopy (FTIR) (Nicolet 6700, Thermo Scientific, Chelmsford, MA, USA) test. CPC, 8.5ALK/CPC, SF/CPC, and 8.5tSF/CPC cements were ground into powders to be characterized by X-ray diffraction (XRD) (X'Pert-Pro MPD, PANalytical, Almelo, the Netherlands).

\section{Mechanical tests}

To evaluate the compression strength of CPCs, the mechanical properties of CPCs, ALK/CPCs, SF/CPCs, and tSF/CPCs were tested using Universal mechanical testing machine (E10000, Instron, Norwood, MA, USA) with 5 repetitions for each group. The cements were hardened and filled in a mold for 1 hour and then ejected into pillar mold with diameter of $6 \mathrm{~mm}$ and height of $12 \mathrm{~mm}$. After that, the

Table I The compositions of different CPCs

\begin{tabular}{lll}
\hline Sample & Composition & Powder \\
\cline { 2 - 3 } & Curing liquid & $\alpha$-TCP \\
\hline CPC & PBS $(\mathrm{pH}=7.4)$ & \\
8ALK/CPC & Treated PBS $(\mathrm{pH}=8.0)$ & \\
8.5ALK/CPC & Treated PBS $(\mathrm{pH}=8.5)$ & \\
9ALK/CPC & Treated PBS $(\mathrm{pH}=9.0)$ & \\
SF/CPC & Untreated SF solution at $\mathrm{pH}$ of 7.4 & \\
8tSF/CPC & Treated SF solution at $\mathrm{pH}$ of 8.0 & \\
8.5tSF/CPC & Treated SF solution at $\mathrm{pH}$ of 8.5 & \\
9tSF/CPC & Treated SF solution at $\mathrm{pH}$ of 9.0 & \\
\hline
\end{tabular}

Abbreviations: ALK, alkaline; CPC, calcium phosphate cement; PBS, phosphate buffer saline; SF, silk fibroin; tSF, SF treated with calcium hydroxide. 
cements were placed at $37^{\circ} \mathrm{C}, 100 \%$ relative humidity for 3 days. After the mechanical tests, the fractured sections of CPCs were characterized by scanning electron microscopy (SEM) (Quanta 250FEG, Thermo Fisher Scientific Electron Microscopy, Hillsboro, OR) and energy dispersive spectrometer (GENESIS, EDAX, Mahwah, NJ).

\section{Handling properties of CPCs}

The handling properties of CPC, 8.5ALK/CPC, SF/CPC, and 8.5tSF/CPC were tested. Both the initial setting time (I-ST) and final setting time (F-ST) were determined at $37^{\circ} \mathrm{C}$ using a Gilmore apparatus, as described previously. ${ }^{28}$ To determine the washout resistance of the cements, they were molded into pallet and then immersed in PBS solution immediately. After that, the appearance of CPCs immersed in PBS at $37^{\circ} \mathrm{C}$ for 20 minutes was recorded by photographing. For injectability test, the CPC was prepared and inserted into a $2 \mathrm{~mL}$ syringe after mixing for 3 minutes, then the paste was ejected from a 11-13G needle and recorded by photographing.

\section{Cell culture}

Extracts from $\mathrm{SF} / \mathrm{CPC}$ and $8.5 \mathrm{tSF} / \mathrm{CPC}$ cements were prepared following the international standard procedure (ISO 10993) for cytotoxicity tests. Briefly, the cements were soaked in alpha-minimum essential medium ( $\alpha$-MEM) solutions (Hyclone, Novato, CA, USA) $\left(3 \mathrm{~cm}^{2} / \mathrm{mL}\right)$ at $37^{\circ} \mathrm{C}$ for 24 hours, then the extracts were collected and sterilized before use. MC3T3-E1 cells (Cell Bank, Shanghai Institutes for Biological Sciences, CAS, Shanghai, China) were seeded at a density of 10,000 cells/well in a 96-well plate. After 4 hours of incubation, the medium was changed into the obtained extracts containing 10\% FBS (Gibco, Grand Island, NY, USA) and 1\% penicillin-streptomycin (Gibco, USA). $\alpha$-MEM medium with no cements immersed was used as control group. The medium was refreshed every 3 days. Cell Counting Kit-8 (CCK-8) tests were performed and the OD at $450 \mathrm{~nm}$ was measured at 1, 3, 5, and 7 days of culture. For cell attachment test, MC3T3-E1 cells were seeded on $8.5 \mathrm{tSF} / \mathrm{CPC}$ plate at a density of $5,000 \mathrm{cells} / \mathrm{cm}^{2}$. After overnight culturing, the cements were dehydrated in graded ethanol and dried in a critical point dryer. The cell morphology on the specimens was imaged by SEM.

\section{Sheep vertebral augmentation tests with CPCs}

The sheep vertebral augmentation test was approved by the Institutional Animal Care and Use Committee of Soochow University. All the experiments were performed following the National Institutes of Health Guide for the Care and Use of Laboratory Animals. In brief, spine specimens (L2-L6) were obtained from fresh female adult small-tail Han sheep with weight range from 40 to $50 \mathrm{~kg}$ provided by the Central Animal Facility of Soochow University. After removing other tissue, a hole $(\Phi=6 \mathrm{~mm})$ was drilled across the middle of the specimen. To make the vertebra upright (slant angle $<1^{\circ}$ ), the top and bottom of vertebrae was covered with PMMA. The drilled vertebrae were considered the defect group, which simulated the osteoporotic vertebrae. In the experimental groups, the drilled vertebrae (in 6 duplicates) were injected with cements to mimic cement-enhanced osteoporotic vertebrae. Six intact specimens and 6 drilled specimens served as blank and control group for mechanical tests. These samples were soaked in PBS for 1 day at $37^{\circ} \mathrm{C}$ before mechanical test (E10000, Instron). In order to eliminate the effects of rheological properties, such as creep and relaxation of the vertebral bodies, it was applied the preload $(200 \mathrm{~N})$ for 30 seconds. The loading rate was $5 \mathrm{~mm} / \mathrm{min}$ during the biomechanical test.

\section{Statistical analysis}

All statistical analyses were performed using SPSS software. Kruskal-Wallis one-way ANOVA tests followed by Tukey post hoc tests were used. Unpaired Student's $t$-tests were also used where appropriate. Difference is considered statistically significant when $P$ is $<0.05$.

\section{Results}

\section{ALK treatment of SF}

SF was treated with saturated ALK, ie, calcium hydroxide, to prepare tSF solutions with various $\mathrm{pH}$. Figure 1 shows

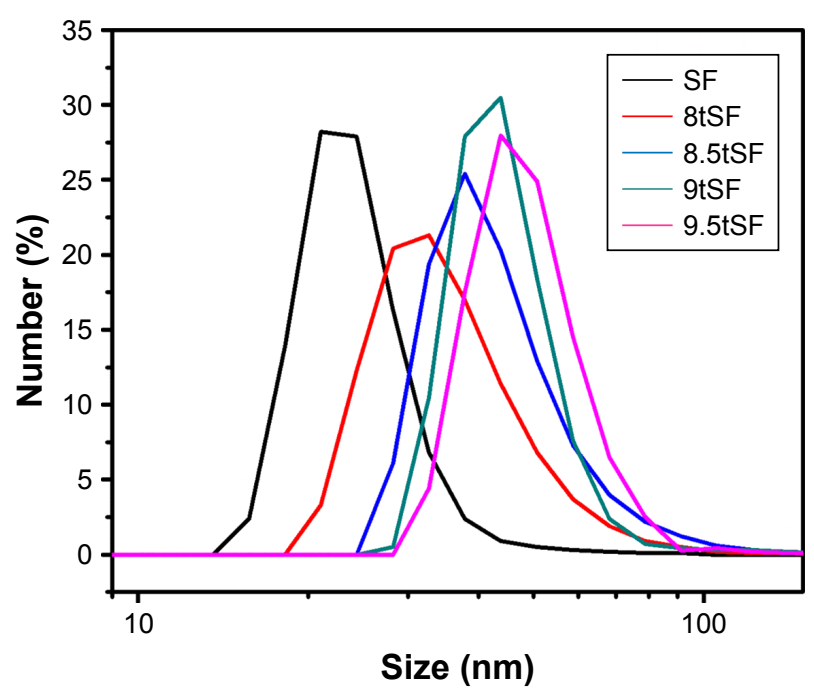

Figure I DLS analysis of 8tSF, 8.5tSF, 9tSF, and 9.5tSF.

Abbreviations: SF, silk fibroin; DLS, dynamic light scattering; tSF, silk fibroin treated with calcium hydroxide. 
Table 2 The zeta potential of different solutions

\begin{tabular}{llllll}
\hline Sample & tSF & 8tSF & $\mathbf{8 . 5 t S F}$ & 9tSF & 9.5tSF \\
\hline Zeta potential $(\mathrm{mV})$ & -1.23 & -7.58 & -15.60 & -10.37 & -5.13 \\
\hline
\end{tabular}

Abbreviation: $t S F$, silk fibroin treated with calcium hydroxide.

that the size of tSF particles increased with $\mathrm{pH}$, being $21,30,40$, and $45 \mathrm{~nm}$ for $8 \mathrm{tSF}, 8.5 \mathrm{tSF}$, 9tSF, and 9.5tSF, respectively. The zeta potential of SF, $8 \mathrm{tSF}, 8.5 \mathrm{tSF}$, 9tSF, and 9.5tSF is shown in Table 2. The zeta potential is $-1.23,-7.58,-15.60,-10.37$, and $-5.13 \mathrm{mV}$ for $\mathrm{SF}, 8 \mathrm{tSF}$, 8.5tSF, 9tSF, and 9.5tSF, respectively. After treatment with calcium hydroxide, more - $\mathrm{COO}-$ and -O- in SF solution was exposed and more negative ions formed. The repulsive force between these negative groups could stretch the SF chain, resulting in a larger particle size. The zeta potential of these particles becomes more negative due to the formation of negative groups with the addition of $\mathrm{Ca}^{2+}$, but negative groups can then bind to $\mathrm{Ca}^{2+}$ ions, contributing to the later decrease in zeta potential. ${ }^{29}$

The FTIR spectra show apparent difference between SF and 8.5tSF (Figure 2). The major difference exists at the 2 strong absorption bands near $1,500 \mathrm{~cm}^{-1}$ and $1,650 \mathrm{~cm}^{-1}$. The bands at $1,515 \mathrm{~cm}^{-1}$ indicate the amide $\mathrm{C}=\mathrm{O}$ bond and the band at $1,625 \mathrm{~cm}^{-1}$ indicates the amide $\mathrm{C}-\mathrm{N}$ bond in the $\mathrm{SF}$ group. These 2 bands indicate the formation of $\beta$-sheets in the secondary structure of SF. However, the amide $\mathrm{C}=\mathrm{O}$ bond and amide $\mathrm{C}-\mathrm{N}$ bond shift to $1,537 \mathrm{~cm}^{-1}$ and $1,648 \mathrm{~cm}^{-1}$, respectively, for tSF, implying a random form of SF chains. ${ }^{30}$ These results indicate that the secondary structure of SF changes after treatment with the calcium hydroxide solution. Compared with SF, the tSF appears to have more random chains rather than $\beta$-sheets.

\section{XRD analysis of CPCs}

The XRD curves of CPC, 8.5ALK/CPC, SF/CPC, and 8.5tSF/CPC cements after 7 days of hardening are shown in Figure 3. The $8.5 \mathrm{tSF} / \mathrm{CPC}$ exhibits obvious HAp peaks at (002), (211, 300, 202, 310, and 222), and (213). ${ }^{23}$ However, peaks of whitlockite $\left(\mathrm{Ca}_{3}\left(\mathrm{PO}_{4}\right)_{2}\right)$ at $2 \theta=27.769^{\circ}, 31.026^{\circ}$, $35.597^{\circ}$, and $41.186^{\circ}$ matched with other CPCs, and whitlockite is generated through the conversion of calcium phosphate from amorphous to crystalline state. ${ }^{24,25}$ This indicates that a fraction of $\alpha$-TCP was not transformed into HAp. Moreover, 8.5tSF/CPC had higher extent of HAp transition than $\mathrm{tSF} / \mathrm{CPC}$.

\section{Mechanical properties of CPCs}

The compressive strength of CPCs was remarkably improved with tSF solution as curing liquid (Figure 4). Specifically, the compressive strength of $8.5 \mathrm{tSF} / \mathrm{CPCs}$ reached $56.3 \pm 1.1 \mathrm{MPa}$. However, all groups using PBS as curing liquid treated with calcium hydroxide solution showed no significant difference from CPCs using PBS. In addition, the compressive strengths are all lower than the SF/CPC groups. The $\mathrm{pH}$ of curing liquid is not the determining factor of the enhancement of SF/CPC. The results indicate that the replacement of PBS with SF solution increases the mechanical property of CPCs. Moreover, the compressive strength could be further improved by treating SF with calcium hydroxide.

The micromorphology of CPCs is shown in SEM images (Figure 5A). CPC exhibits many plate-like crystals with some large grains. Many needle-like crystals with large grains were also found in the ALK/CPC group. However, the crystals
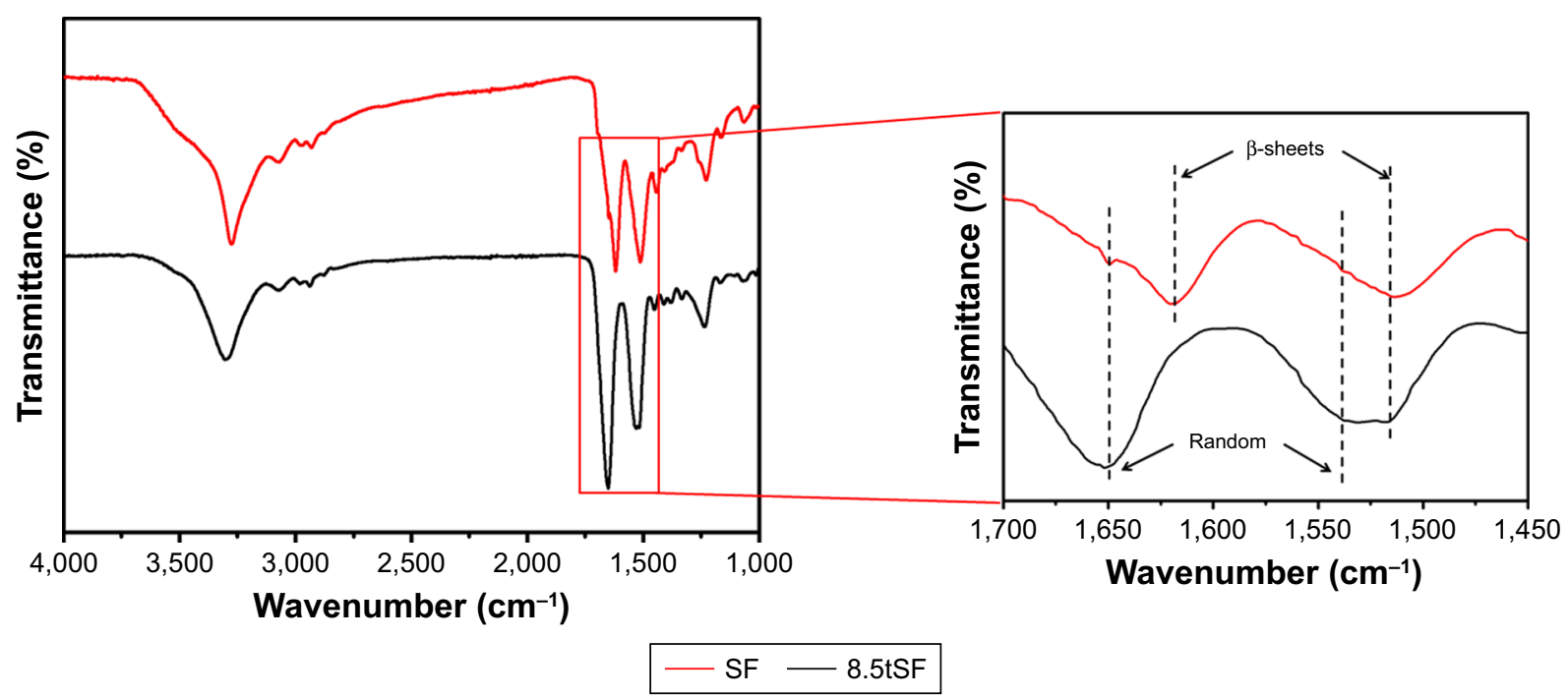

Figure 2 FTIR spectra of SF and 8.5tSF.

Abbreviations: FITR, Fourier transform infrared spectroscopy; tSF, silk fibroin treated with calcium hydroxide; SF, silk fibroin. 


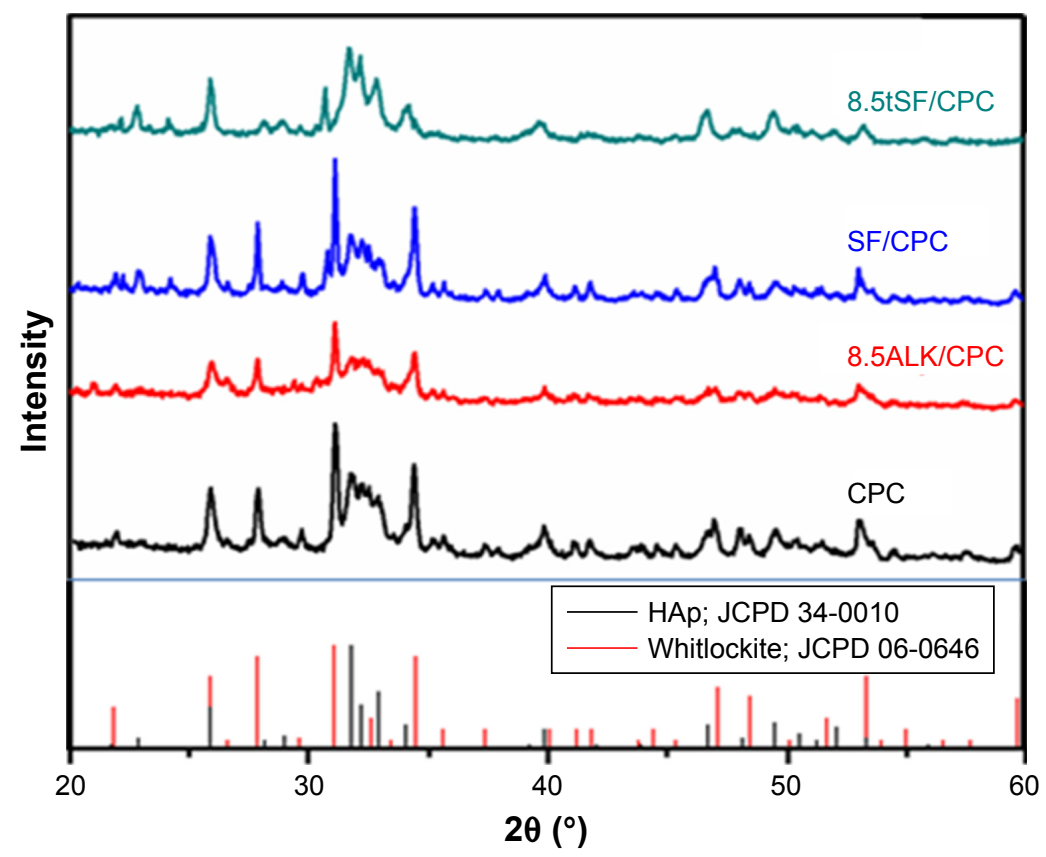

Figure 3 XRD patterns of CPC, 8.5ALK/CPC, SF/CPC, and 8.5tSF/CPC.

Abbreviations: ALK, alkaline; CPC, calcium phosphate cement; HAp, hydroxyapatite; tSF, silk fibroin treated with calcium hydroxide; XRD, X-ray diffraction; SF, silk fibroin.

are surrounded by a mass of clusters for $\mathrm{SF} / \mathrm{CPC}$ when $\mathrm{SF}$ is added. Plate-like crystals are found in SF/CPC, but more needle-like in $\mathrm{tSF} / \mathrm{CPC}$. The clusters in $\mathrm{tSF} / \mathrm{CPC}$ are closer than in SF/CPC. Figure 5B demonstrates that higher carbon content is found in the clusters and the amount is much greater than in the crystals. According to the results, the clusters are more similar to a mixture of SF and CPCs.

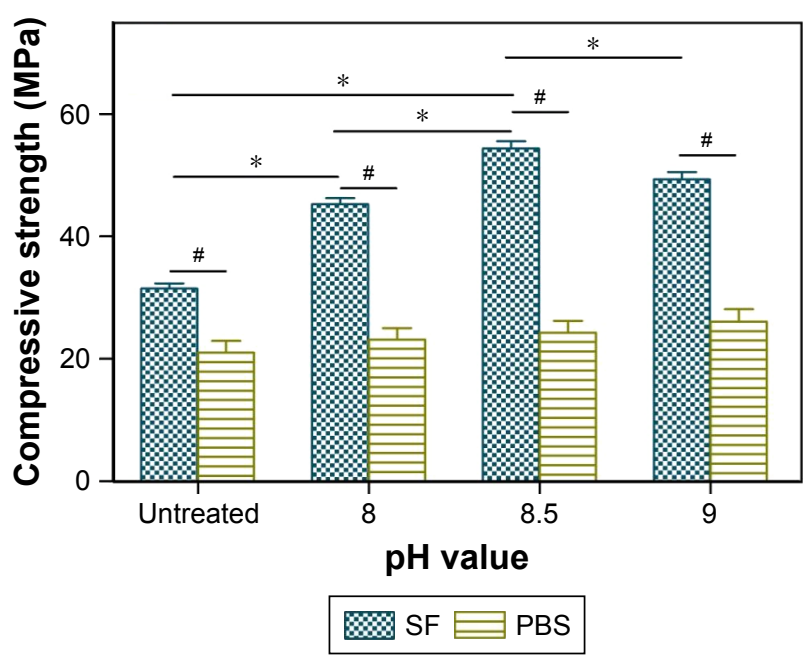

Figure 4 The compressive strength of CPCs using PBS and SF with different $\mathrm{pH}$ values as curing liquids.

Notes: *, significant difference between the $\mathrm{pH}$ value using $\mathrm{SF}$ as curing agent. $(P<0.05) .{ }^{*}$, significant difference between the SF and $\mathrm{PBS}$ at the same $\mathrm{pH}$ value $(P<0.05)$.

Abbreviations: CPC, calcium phosphate cement; PBS, phosphate buffer saline; SF, silk fibroin.

\section{Handling properties of CPCs}

Based on the mechanical test results, tSF/CPC possesses the highest compressive strength and ALK/CPC, which has the same $\mathrm{pH}$ as $\mathrm{tSF} / \mathrm{CPC}$, was selected to test the handling properties of cements. The setting times of different bone cements are summarized in Table 3. The setting time of $8.5 \mathrm{tSF} / \mathrm{CPC}$ (I-ST, $24.53 \pm 0.88$ minutes; F-ST, $41.03 \pm 1.06$ minutes) is much shorter than that of CPC (I-ST, $41.67 \pm 1.32$ minutes; F-ST, $62.04 \pm 1.66$ minutes) and $8.5 \mathrm{ALK}$ CPC (I-ST, $34.23 \pm 1.55$ minutes; F-ST, $54.23 \pm 1.78$ minutes), but longer than SF/CPC (I-ST, $17.69 \pm 1.72$ minutes; F-ST, $32.00 \pm 0.95$ minutes) in both the initial and final settings. Clearly, the setting time was prolonged in ALK condition. This is because the hydration reaction slowed down when more $\mathrm{OH}^{-}$was added, which retarded the solidification process. However, with the addition of SF, the setting time could be shortened, likely because that SF might act as an adhesive network to solidify CPC and prevent CPC particles from moving around. In the anti-washout tests, the CPC paste collapsed into powder and could not maintain the pellet shape; ALK/CPC paste also collapsed but still maintained the pellet shape, SF/CPC paste showed peeling edges and strongly maintained the pallet shape, but no collapse was observed in tSF/CPC (Figure 6A). The $8.5 \mathrm{tSF} / \mathrm{CPC}$ still possessed decent injectability. It could be easily ejected from a syringe and well maintained the original shape in PBS (Figure 6B). In addition, it could also be injected from 

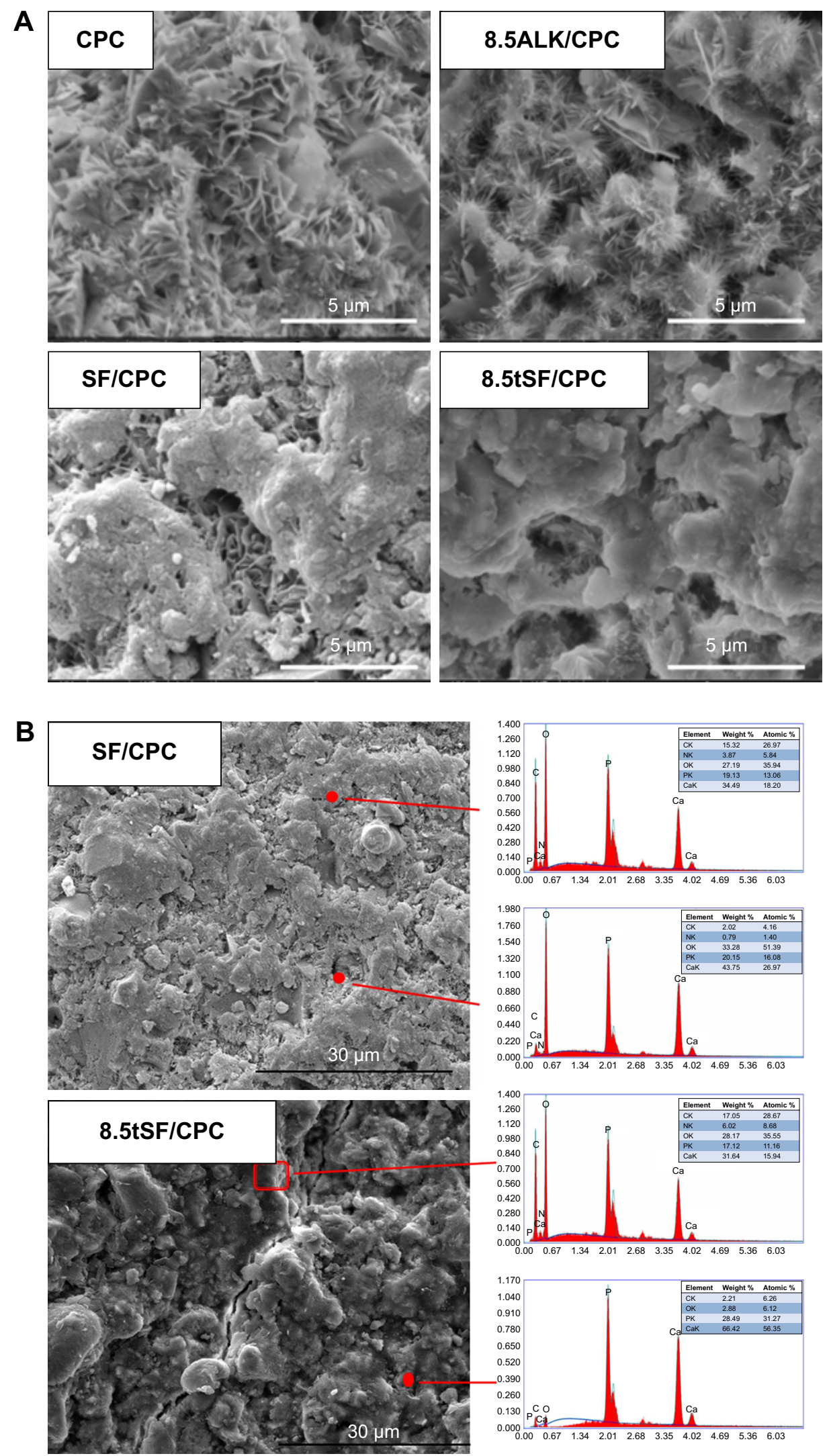

Figure 5 (A) SEM images of CPC, 8.5ALK/CPC, SF/CPC, and 8.5tSF/CPC. (B) SEM images and EDS spectra of SF/CPC and 8.5tSF/CPC. Scale bars: $5 \mu \mathrm{m}$ (A) and $30 \mu \mathrm{m}$ (B). Abbreviations: ALK, alkaline; CPC, calcium phosphate cement; EDS, energy dispersive spectrometer; SEM, scanning electron microscopy; SF, silk fibroin; tSF, silk fibroin treated with calcium hydroxide; XRD, X-ray diffraction. 
Table 3 The initial and final setting times of CPCs

\begin{tabular}{lllll}
\hline Sample & CPC & 8.5ALK/CPC & SF/CPC & 8.5tSF/CPC \\
\hline $\begin{array}{l}\text { Initial setting } \\
\text { time (min) }\end{array}$ & $41.67 \pm 1.32$ & $34.23 \pm 1.55$ & $17.69 \pm 1.72$ & $24.53 \pm 0.88$ \\
$\begin{array}{l}\text { Final setting } \\
\text { time (min) }\end{array}$ & $62.04 \pm 1.66$ & $54.23 \pm 1.78$ & $32.00 \pm 0.95$ & $41.03 \pm 1.06$ \\
\hline
\end{tabular}

Abbreviations: ALK, alkaline; CPC, calcium phosphate cement; SF, silk fibroin; tSF, silk fibroin treated with calcium hydroxide.

11 to $13 \mathrm{G}$ needle. All these results indicate that $8.5 \mathrm{tSF} /$ $\mathrm{CPC}$ may be an ideal candidate filler material for $\mathrm{VP} / \mathrm{KP}$ surgeries. $^{25}$

\section{Biocompatibility of CPCs}

There was no difference in the CCK-8 results observed between the control group, SF/CPC and $8.5 \mathrm{tSF} / \mathrm{CPC}$, and as shown in Figure 7A, the OD value of all 3 groups increased in a stable manner on day 1 to day 5 . This result showed that both SF/CPC and 8.5tSF/CPC have low cytotoxicity. The MC3T3-E1 cell can attach and grow on the surface of the 8.5tSF/CPC cements from the SEM images in Figure 7B. The attached cells showed good morphology and stretch on the surface of $8.5 \mathrm{tSF} / \mathrm{CPC}$.

\section{Sheep vertebra augmentation tests with CPCs}

The mechanical properties of CPC-filled sheep vertebrae are shown in Figure 8. Compared with those of drilled vertebrae, both the compressive strength and stiffness of CPC-filled vertebrae were increased. The replacement of CPC with $\mathrm{SF} / \mathrm{CPC}$ also contributes to higher mechanical properties. The 8.5tSF/CPC group showed the highest compressive strength at 20.02 $\pm 1.73 \mathrm{MPa}$ and stiffness at $2.75 \pm 0.47 \mathrm{~N} / \mathrm{mm}$, which were comparable with those of native vertebrae.

\section{Discussion}

CPCs have been studied for decades since it was invented. Due to the limited mechanical properties, they are usually used for repairing defects of nonload-bearing bones, such as radius,${ }^{44}$ or for load-bearing bone repair in combination with fixation systems. ${ }^{44}$ They cannot be used alone in minimally invasive spine surgeries, such as VP and KP. ${ }^{45}$ Recently, increasing studies have composited CPCs with other materials to enhance their mechanical and handling properties. Among these materials, polymers, both natural and synthetic, are attracting increasing interest. ${ }^{31-33,46}$ The structure and functional groups of polymers dramatically impact the mechanical properties of CPC composites. Taking advantage of the massive functional groups, such as $-\mathrm{COOH}$ and $-\mathrm{OH}$ in its molecular chain, SF has been composited with CPCs in many studies. The compressive strength of CPC/SF composites has been reported to be as high as $50 \mathrm{MPa} .{ }^{24,25}$ However, CPC-based composite cements remain to be improved so that they are comparable with PMMA cements, whose compressive strength exceeds $70 \mathrm{MPa}$.
A
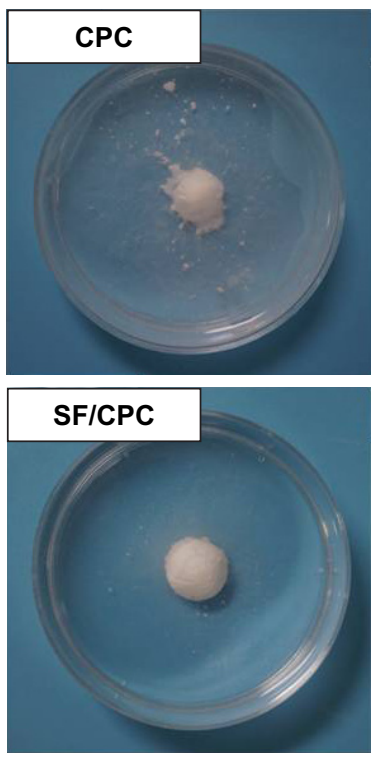
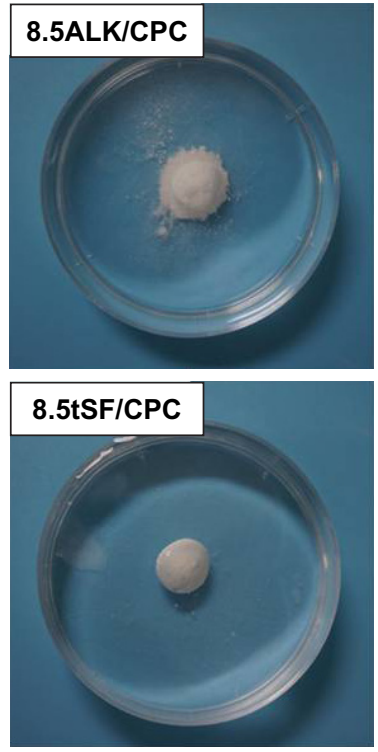

B

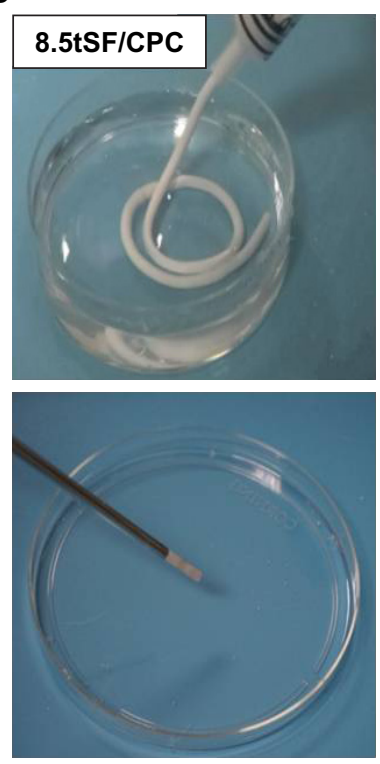

Figure 6 The anti-washout property (A) of different CPCs and injectable performance (B) of 8.5tSF/CPC. Abbreviations: ALK, alkaline; CPC, calcium phosphate cement; SF, silk fibroin; tSF, silk fibroin treated with calcium hydroxide. 


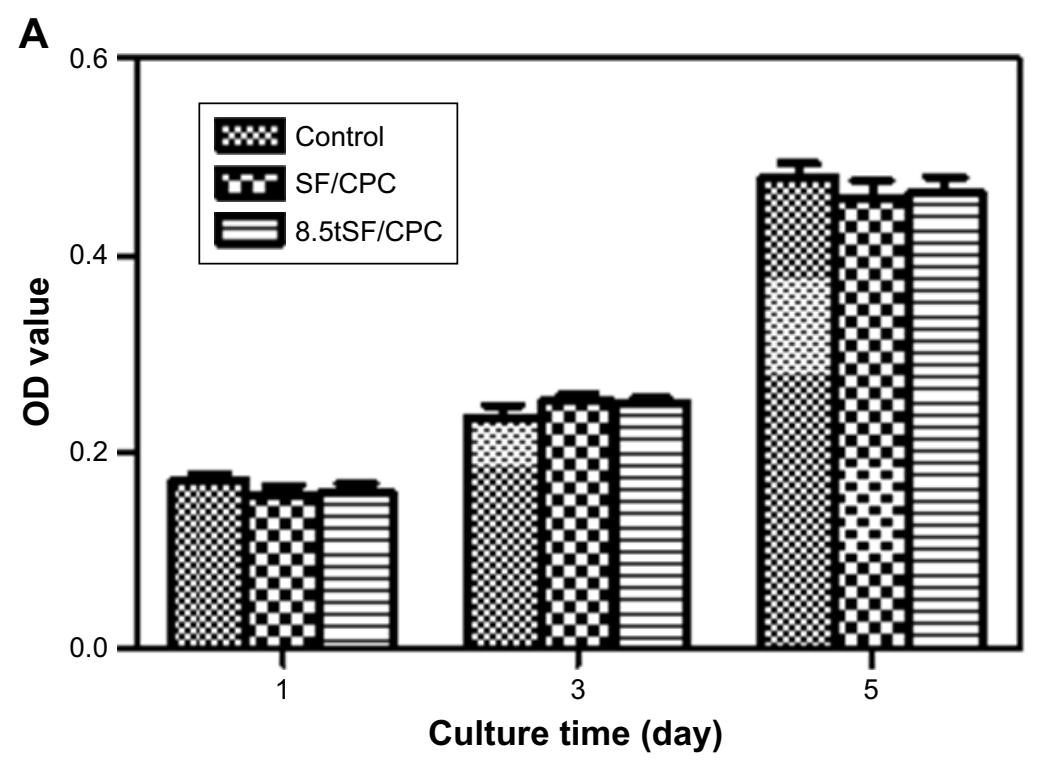

B

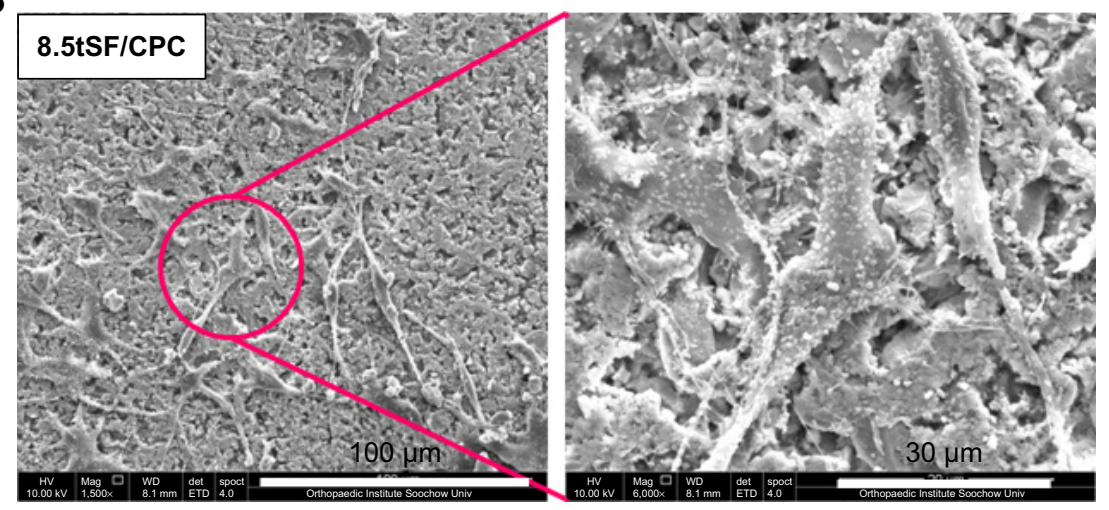

Figure 7 (A) Proliferation of MC3T3-EI cell on different bone cements. (B) SEM images of MC3T3-EI cell adhesion on 8.5tSF/CPC bone cement.

Abbreviations: CPC, calcium phosphate cement; SF, silk fibroin; OD, optical density; SEM, scanning electron microscopy; tSF, silk fibroin treated with calcium hydroxide.

It has been reported that the interfacial binding between organic and inorganic phases in composited CPCs could be enhanced by introducing functional groups to the additives. Since the structure and surface charge of SF is affected by the $\mathrm{pH}$ of solution, ${ }^{34,35}$ in this study, we treated SF with calcium hydroxide to obtain tSF with different structures and surface

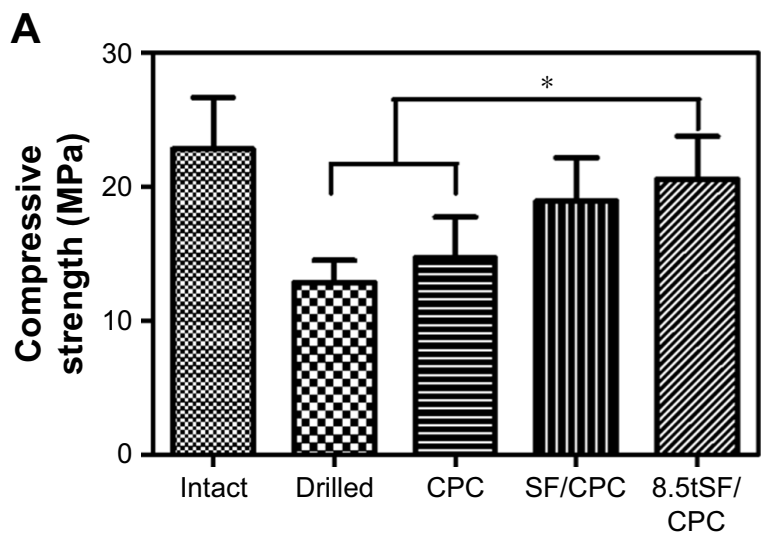

charges so that it could be used as a reinforcing additive in CPCs. We found that particle size of SF increased with the $\mathrm{pH}$ of solution (Figure 1). In contrast, the zeta potential of SF solutions showed different behaviors. The charges of the SF chains became more negative when $\mathrm{pH}$ increased from 7 to 8.5 and then decreased. These results imply that with the

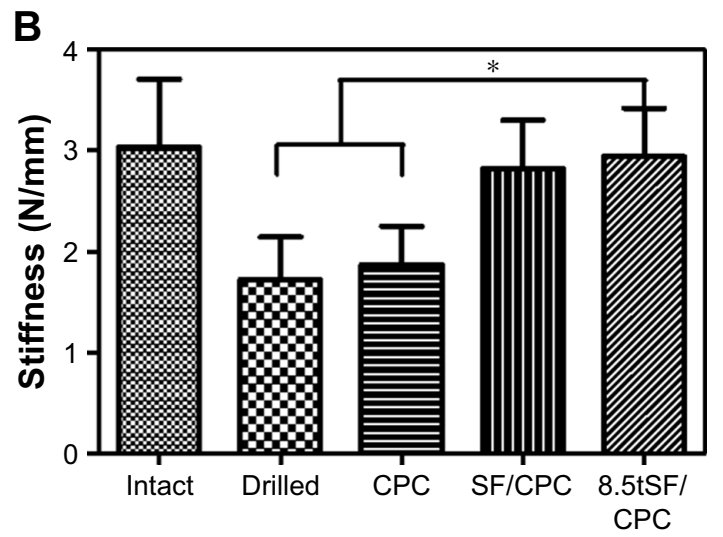

Figure 8 The compressive strength (A) and stiffness $(\mathbf{B})$ of intact, drilled, CPC, SF/CPC and 8.5tSF/CPC filled vertebrae. $* P<0.05$. Abbreviations: CPC, calcium phosphate cement; SF, silk fibroin; tSF, silk fibroin treated with calcium hydroxide. 
addition of calcium hydroxide, the SF chains first began to carry more negative charge and grew larger. However, as the content of calcium hydroxide increased, the superfluous $\mathrm{Ca}^{+}$ ions blocked negative charges. ${ }^{34}$ In addition, more random SF chains were found after they were treated with calcium hydroxide, which may imply a more stretched morphology of $\mathrm{SF}$ chains in $\mathrm{tSF}$ solution. These results agree with previous studies about the conformation of SF under ALK conditions. ${ }^{36}$ Hence, tSF solutions with more random SF chains and negative charges were used in our next study.

The low mechanical strength of CPCs is a major problem to be solved. Previously, reinforcing agents, such as carbon nanotubes/HAp composites were added to CPCs for mechanical reinforcement. ${ }^{14}$ In a report, acid-modified SF was used to reinforce magnesium phosphate cements (MPCs). ${ }^{24}$ With this method, the mechanical property of MPCs improved, since the interfacial combination between SF and MPCs was enhanced. In our study, ALK-treated SF solution was used as curing liquid to prepare CPCs. When tSF is composited with $\alpha-\mathrm{TCP}$, significant enhancement is obtained, with the compressive strength reaching as high as $56.3 \pm 1.1 \mathrm{MPa}$. In addition, the compressive strength of CPCs increased with $\mathrm{pH}$, proving that $\mathrm{tSF}$ could indeed improve the mechanical property of CPCs. However, for the control group with PBS solution as curing liquid, regardless of $\mathrm{pH}$, the compressive strength was not significantly different and was indeed significantly lower than that of SF groups. Based on the zeta potential and dynamic light scattering results, the more negative charges and more stretched structure of SF might play critical roles in this reinforcing effect. These negative charges might originate from the hydrolyzed $-\mathrm{COOH}$ groups on SF chains, which could fortify the interfacial interactions between SF and $\alpha-T C P$, and thereby improve the mechanical property of the latter.

Further, XRD analysis results show that a stronger HAp peak exists in the spectrum of $\mathrm{tSF} / \mathrm{CPC}$ instead of other 3 groups, indicating high extent of HAp transition in this group. As reported in a previous study, high HAp transition implies more complete CPC hydration and results in better formation of HAp crystals and more stable structure of CPCs. ${ }^{41}$ This appears also true in our study, as can be seen from the morphology of $\mathrm{tSF} / \mathrm{CPC}$, which shows a compact structure with many clusters and needle-like crystals (Figure 5). The clusters were also found in SF/CPC groups. In addition to $\mathrm{Ca}$ and $\mathrm{P}$ elements, a massive $\mathrm{C}$ element exists in the clusters, which indicate that this cluster could be a mixture of SF and CPCs. In the ALK condition, the flowerlike crystal changes to a needle-like form. This phenomenon echoes the findings in the study of Tanahashi et al, in which needle-like HAp crystals were observed in $\alpha$-TCP cured in an ALK environment $(\mathrm{pH}=10) .{ }^{42}$ In our study, the needlelike crystal section occupied less space than the flower-like crystal section in $\mathrm{tSF} / \mathrm{CPC}$, resulting in fewer microgaps and more compact morphology of CPC than those in other groups, which strengthened the CPCs. ${ }^{43}$ Using sheep vertebra as a model for vertebra augmentation tests, we found that tSF/CPC-filled vertebrae had highest mechanical properties compared with other CPC-filled ones. Moreover, the vertebrae filled with $\mathrm{tSF} / \mathrm{CPC}$ showed no statistical difference with native vertebrae, suggesting that $\mathrm{tSF} / \mathrm{CPC}$ may be a promising load-bearing filler material for vertebral augmentation. The similar stiffness between $\mathrm{tSF} / \mathrm{CPC}$-filled vertebrae and intact vertebrae demonstrated that the filling material in the vertebra could play the same role in mechanical function. It should be noted that while mechanical recovery has been reached by $\mathrm{tSF} / \mathrm{CPC}$ injection, there is still potential risk from the stiffness mismatch between tSF/CPC and local tissue of the vertebra. According to a previous study, the relative higher stiffness of filling material within vertebrae could affect the microphysiological activity of them and might result in new vertebra fracture or deterioration. ${ }^{28}$ Therefore, other effects of tSF/CPC-augmented vertebrae should also be checked in our further study.

The handling property is another concern for clinical applications of CPCs. The setting times and anti-washout property of currently used CPCs are inferior compared with conventional PMMA bone cements. ${ }^{37}$ Ideally, the I-ST should be no $<5$ minutes and the F-ST should be no $>30$ minutes. ${ }^{25}$ In our work, the hardening time will be shorter when $\mathrm{CPC}$ were mixed with SF. Using ALK-treated SF as a curing liquid could further reduce the hardening time. It is known that hydration of $\alpha$-TCP is more favorable in an ALK condition, where abundant HAp is produced and adhere to HAp nuclear sites to continue growing. ${ }^{38}$ This process may accelerate the hardening of CPCs. As has been previously reported, polymers, such as chitosan and silk could shorten the setting time of CPCs when composited with them. ${ }^{26}$ However, in our study tSF indeed slowed down the hardening process compared with untreated SF. This might be attributed to the special rheological property of tSF solution, which had lower viscosity compared with untreated SF solution. ${ }^{35}$ The water in SF solution with lower viscosity could fast diffuse in mixed CPCs paste and accelerate the hydration process during the initial setting and hardening periods. As cement leakage can result in severe complications, such as fatal pulmonary embolism, the anti-washout property is another important factor and greatly affects the clinical applications of CPCs. ${ }^{39} \mathrm{We}$ found that the anti-washout property of CPCs 
was markedly improved by tSF. This is in agreement with an earlier study, in which addition of chitosan was able to prevent CPCs from collapse in water. ${ }^{40}$

\section{Conclusion}

In this study, composite CPCs have been prepared using $\alpha$-TCP powder and $\mathrm{tSF}$, that is, calcium hydroxide-treated SF solution. The tSF/CPC cement showed significantly higher compressive strength than CPC. Particularly, 8.5tSF/CPC, that is composite of $\alpha$-TCP and tSF at $\mathrm{pH}$ of 8.5 , showed the highest compressive strength $(56.3 \pm 1.1 \mathrm{MPa})$. The enhancement of CPCs may be attributed to the following 2 facts. First, tSF might enhance the interfacial bonding between SF, the organic phase, and CPC, the inorganic phase of composite cement. Second, the conversion rate of HAp from $\alpha$-TCP was also improved by tSF. The $\mathrm{tSF} / \mathrm{CPC}$ showed considerable biocompatibility in vitro. The drilled sheep vertebrae filled with $\mathrm{tSF} / \mathrm{CPC}$ possessed comparable mechanical properties as native vertebrae. Therefore, calcium hydroxide-treated SF solution as curing liquid may be an effective approach to enhance the mechanical properties of CPCs. However, the stiffness of $\mathrm{tSF} / \mathrm{CPC}$ composite cement is still 1 limitation for its application in VP/KP. The curing time should also be optimized to meet the clinical requirement. Morever, the in vivo effects of $\mathrm{tSF} / \mathrm{CPC}$ are to be evaluated. Our following study will address these issues.

\section{Acknowledgments}

The authors are grateful to the funding support from the National Natural Science Foundation of China (81471790, 31500779, 81672213, 81772358), Jiangsu Provincial Special Program of Medical Science (BL2012004), China Postdoctoral Science Foundation (2016M590500, 2017T100398), Jiangsu Planned Projects for Postdoctoral Research Funds (1601269C), the Priority Academic Program Development, and the Natural Science Foundation of Jiangsu Higher Education Institutions of China (15KJB310021).

\section{Author contributions}

$\mathrm{MH}, \mathrm{ZH}, \mathrm{PZ}$, and FL performed all the experiments. XZ and HY supervised the ex vivo experiments. $\mathrm{MH}, \mathrm{FH}, \mathrm{CS}$, and $\mathrm{BL}$ analyzed the data and wrote the manuscript. All authors reviewed and approved the final version of manuscript. All authors contributed to data analysis, drafting or revising the article, gave final approval of the version to be published, and agree to be accountable for all aspects of the work.

\section{Disclosure}

The authors declare no conflicts of interest with this work.

\section{References}

1. He Z, Zhai Q, Hu M, et al. Bone cements for percutaneous vertebroplasty and balloon kyphoplasty: current status and future developments. J Orthop Translat. 2015;3(1):1-11.

2. Zhang J, Liu W, Schnitzler V, Tancret F, Bouler JM. Calcium phosphate cements for bone substitution: chemistry, handling and mechanical properties. Acta Biomater. 2014;10(3):1035-1049.

3. Geffers M, Groll J, Gbureck U. Reinforcement strategies for load-bearing calcium phosphate biocements. Materials. 2015;8(5):2700-2717.

4. del Real RP, Wolke JG, Vallet-Regí M, Jansen JA. A new method to produce macropores in calcium phosphate cements. Biomaterials. 2002; 23(17):3673-3680.

5. Vorndran E, Geffers M, Ewald A, Lemm M, Nies B, Gbureck U. Ready-to-use injectable calcium phosphate bone cement paste as drug carrier. Acta Biomater. 2013;9(12):9558-9567.

6. Lopez-Heredia MA, Sariibrahimoglu K, Yang W, et al. Influence of the pore generator on the evolution of the mechanical properties and the porosity and interconnectivity of a calcium phosphate cement. Acta Biomater. 2012;8(1):404-414.

7. Barralet JE, Hofmann M, Grover LM, Gbureck U. High-strength apatitic cement by modification with $\alpha$-hydroxy acid salts. Adv Mater. 2003; 15(24):2091-2094.

8. Wang J, Liu C, Liu Y, Zhang S. Double-network interpenetrating bone cement via in situ hybridization protocol. Adv Funct Mater. 2010; 20(22):3997-4011

9. Hh X, Quinn JB, Takagi S, Chow LC, Eichmiller FC. Strong and macroporous calcium phosphate cement: effects of porosity and fiber reinforcement on mechanical properties. J Biomed Mater Res. 2015; 57(3):457-466.

10. Xu HH, Quinn JB. Calcium phosphate cement containing resorbable fibers for short-term reinforcement and macroporosity. Biomaterials. 2002;23(1):193-202.

11. Qi X, Ye J, Wang Y. Improved injectability and in vitro degradation of a calcium phosphate cement containing poly(lactide-co-glycolide) microspheres. Acta Biomater. 2008;4(6):1837-1845.

12. Tian Q, Jing H, Zhang M, et at. Effects of adding carbon nanotubes/ hydroxyapatite composites on the properties and structure of calcium phosphate cement. J Inorg Mater. 2013;28(1):91-96.

13. Aryaei A, Liu J, Jayatissa AH, Jayasuriya AC. Cross-linked chitosan improves the mechanical properties of calcium phosphate-chitosan cement. Mater Sci Eng C Mater Biol Appl. 2015;54:14-19.

14. Yokoyama A, Yamamoto S, Kawasaki T, Kohgo T, Nakasu M. Development of calcium phosphate cement using chitosan and citric acid for bone substitute materials. Biomaterials. 2002;23(4):1091-1101.

15. Liu W, Zhang J, Weiss P, Tancret F, Bouler JM. The influence of different cellulose ethers on both the handling and mechanical properties of calcium phosphate cements for bone substitution. Acta Biomater. 2013;9(3):5740-5750.

16. Christel T, Kuhlmann M, Vorndran E, Groll J, Gbureck U. Dual setting $\alpha$-tricalcium phosphate cements. J Mater Sci Mater Med. 2013;24(3): 573-581.

17. Maenz S, Hennig M, Mühlstädt M, et al. Effects of oxygen plasma treatment on interfacial shear strength and post-peak residual strength of a PLGA fiber-reinforced brushite cement. J Mech Behav Biomed Mater. 2016;57:347-358.

18. Low KL, Zein SHS, Tan SH, Mcphail DS, Boccaccini AR. The effect of interfacial bonding of calcium phosphate cements containing biomineralized multi-walled carbon nanotube and bovine serum albumin on the mechanical properties of calcium phosphate cements. Ceram Int. 2011;37(7):2429-2435. 
19. Vepari C, Kaplan DL. Silk as a biomaterial. Prog Polym Sci. 2007; 32(8-9):991-1007.

20. Wang B, Xie RJ, Huang YY. Preparation and characterization of silk fibroin/ calcium phosphate composite. Adv Mater Rese. 2011; Vols. 332-334: $1655-1658$.

21. Ding T, Yang H, Maltenfort M, Xie R. Silk fibroin added to calcium phosphate cement to prevent severe cardiovascular complications. Med Sci Monit. 2010;16(9):HY23-HY26.

22. Li S, Liu B, Cheng J, Hu J. Composite cement of magnesium-bearing phosphoaluminate-hydroxyapatite reinforced by treated raw silk fiber. Cement Concrete Comp. 2008;30(4):347-352.

23. Cao C, Li H, Li J, Liu C, Yang H, Li B. Mechanical reinforcement of injectable calcium phosphate cement/silk fibroin (SF) composite by mineralized SF. Ceram Int. 2014;40(9):13987-13993.

24. Feng T, Pi B, Li B, et al. N-Acetyl cysteine (NAC)-mediated reinforcement of alpha-tricalcium phosphate/silk fibroin $(\alpha-\mathrm{TCP} / \mathrm{SF})$ cement. Colloids Surf B Biointerfaces. 2015;136:892-899.

25. Mandal BB, Grinberg A, Gil ES, Panilaitis B, Kaplan DL. High-strength silk protein scaffolds for bone repair. Proc Natl Acad Sci U S A. 2012; 109(20):7699-7704.

26. Zhu X, Chen X, Chen C, et al. Evaluation of calcium phosphate and calcium sulfate as injectable bone cements in sheep vertebrae. J Spinal Disord Tech. 2012;25(6):333-337.

27. Zhou P, Xie X, Knight DP, Zong XH, Deng F, Yao WH. Effects of $\mathrm{pH}$ and calcium ions on the conformational transitions in silk fibroin using 2D Raman correlation spectroscopy and 13C solid-state NMR. Biochemistry. 2004;43(35):11302-11311.

28. Aksakal B, Koç K, Yargı Ö, Tsobkallo K. Effect of UV-light on the uniaxial tensile properties and structure of uncoated and $\mathrm{TiO} 2$ coated Bombyx mori silk fibers. Spectrochim Acta A Mol Biomol Spectrosc. 2016;152:658-665.

29. Wang X, Ma J, Wang Y, He B. Structural characterization of phosphorylated chitosan and their applications as effective additives of calcium phosphate cements. Biomaterials. 2001;22(16):2247-2255.

30. Gallinetti S, Mestres G, Canal C, Persson C, Ginebra MP. A novel strategy to enhance interfacial adhesion in fiber-reinforced calcium phosphate cement. J Mech Behav Biomed Mater. 2017;75:495-503.

31. Castro AGB, Polini A, Azami Z, et al. Incorporation of PLLA microfillers for mechanical reinforcement of calcium-phosphate cement. J Mech Behav Biomed Mater. 2017;71:286-294.

32. Terry AE, Knight DP, Porter D, et al. $\mathrm{pH}$ induced changes in the rheology of silk fibroin solution from the middle division of Bombyx mori silkworm. Biomacromolecules. 2004;5(3):768-772.

33. Matsumoto A, Lindsay A, Abedian B, Kaplan DL. Silk fibroin solution properties related to assembly and structure. Macromol Biosci. 2008; 8(11):1006-1018.
34. Gotoh Y, Tsukada M, Minoura N. Chemical modification of the arginyl residue in silk fibroin: 2 . Reaction of 1,2-cyclohexanedione in aqueous alkaline medium. Int J Biol Macromol. 1996;19(1):41-44.

35. Sugawara A, Asaoka K, Ding S-J. Calcium phosphate-based cements: clinical needs and recent progress. J Mater Chem B. 2013;1(8): 1081-1089.

36. Park HC, Baek DJ, Park YM, Yoon SY, Stevens R. Thermal stability of hydroxyapatite whiskers derived from the hydrolysis of alpha-TCP. J Mater Sci. 2004;39(7):2531-2534.

37. Krebs J, Aebli N, Goss BG, et al. Cardiovascular changes after pulmonary embolism from injecting calcium phosphate cement. J Biomed Mater Res B Appl Biomater. 2007;82(2):526-532.

38. Takechi M, Miyamoto Y, Ishikawa K, et al. Non-decay type fast-setting calcium phosphate cement using chitosan. J Mater Sci Mater Med. 1996;7(6):317-322.

39. Bimis A, Karalekas D, Bouropoulos N, Mouzakis D, Zaoutsos S. Monitoring of hardening and hygroscopic induced strains in a calcium phosphate bone cement using FBG sensor. J Mech Behav Biomed Mater. 2016;60:195-202.

40. Tanahashi M, Kamiya K, Suzuki T, Nasu H. Fibrous hydroxyapatite grown in the gel system: effects of $\mathrm{pH}$ of the solution on the growth rate and morphology. J Mater Sci Mater Med. 1992;3(1):48-53.

41. Yu T, Ye J, Wang Y. Preparation and characterization of a novel strontium-containing calcium phosphate cement with the two-step hydration process. Acta Biomater. 2009;5(7):2717-2727.

42. Jones GL, Motta A, Marshall MJ, El Haj AJ, Cartmell SH. Osteoblast: osteoclast co-cultures on silk fibroin, chitosan and PLLA films. Biomaterials. 2009;30(29):5376-5384.

43. Kopylov P, Runnqvist K, Jonsson K, Aspenberg P. Norian SRS versus external fixation in redisplaced distal radial fractures. A randomized study in 40 patients. Acta Orthop Scand. 1999;70(1):1-5.

44. Wagoner Johnson AJ, Herschler BA. A review of the mechanical behavior of $\mathrm{CaP}$ and $\mathrm{CaP} /$ polymer composites for applications in bone replacement and repair. Acta Biomater. 2011;7(1):16-30.

45. Zhang B, Dai M. Filler materials used in kyphoplasty and vertebroplasty for osteoporotic vertebral compression fractures. Adv Mater Res. 2012 Vols. 393-395:776-771.

46. Andreasen CM, Henriksen SS, Ding M, Theilgaard N, Andersen TL, Overgaard S. The efficacy of poly-d,l-lactic acid- and hyaluronic acidcoated bone substitutes on implant fixation in sheep. J Orthop Translat. 2017;8(1):12-19.
International Journal of Nanomedicine

\section{Publish your work in this journal}

The International Journal of Nanomedicine is an international, peerreviewed journal focusing on the application of nanotechnology in diagnostics, therapeutics, and drug delivery systems throughout the biomedical field. This journal is indexed on PubMed Central, MedLine, CAS, SciSearch ${ }^{\circledR}$, Current Contents ${ }^{\circledR} /$ Clinical Medicine,

\section{Dovepress}

Journal Citation Reports/Science Edition, EMBase, Scopus and the Elsevier Bibliographic databases. The manuscript management system is completely online and includes a very quick and fair peer-review system, which is all easy to use. Visit http://www.dovepress.com/ testimonials.php to read real quotes from published authors. 